\title{
CORRIGENDUM TO "THE IDEAL THEORY IN QUOTIENTS OF COMMUTATIVE SEMIRINGS"
}

\author{
Shahabaddin Ebrahimi Atani, Saboura Dolati Pish Hesari and \\ MEHDi KHORAMDEL \\ University of Guilan, Iran
}

\begin{abstract}
This corrigendum is written to correct an error in the proof of the Theorem 2.16 of S. E. Atani [1].
\end{abstract}

The subject of this paper is to correct Theorem 2.16 of [1]. It reads as follows.

Theorem. Let I be a $Q$-ideal of a semiring $R$. Then the following hold:

(i) if both $I$ and $R / I$ are Noetherian, then $R$ is Noetherian,

(ii) if both $I$ and $R / I$ are Artinian, then $R$ is Artinian.

In the proof of this theorem in [1] it was used that the sum $I+J$ of two k-ideals $I$ (note that any $Q$-ideal is a k-ideal, but not conversely) and $J$ of $R$ is again a k-ideal. Since it is well known that this is not true in general, this theorem is not true either. But in the two following special cases, the proof and the theorem (under each of these additional assumptions) remain valid.

a) $R$ is a $k$-semiring, i.e. every ideal of $R$ is a k-ideal.

b) $I$ is a strong $k$-ideal of $R$, i.e. for every $a \in I$ there is some $b \in I$ such that $a+b=0$.

Since case a) is obvious, it is sufficient to show that in case b) the sum $I+J$ for every k-ideal $J$ of $R$ is also a k-ideal. But if $a+j, a+j+x=a^{\prime}+j^{\prime} \in I+J$ for $a, a^{\prime} \in I, j, j^{\prime} \in J$ and $x \in R$, there is some $b^{\prime} \in I$ such that $a^{\prime}+b^{\prime}=0$, since $I$ is strong. This implies $j^{\prime}=a^{\prime}+b^{\prime}+j^{\prime}=a+b^{\prime}+j+x \in J$, hence $a+b^{\prime}+x \in J$, since $J$ is a k-ideal of $R$. Now, since $a+b^{\prime} \in I$, there is some $c \in I$ such that $a+b^{\prime}+c=0$. Therefore $x=c+a+b^{\prime}+x \in I+J$, and so $I+J$ is a k-ideal of $R$.

2010 Mathematics Subject Classification. 16Y60.

$K e y$ words and phrases. Semiring, $k$-ideals, $Q$-ideals, quotient semiring. 
Clearly, Lemma 2.12 (i) and Lemma 2.13 (i) in [1] have to be deleted.

ACKNOWLEDGEMENTS.

The authors express their deep gratitude to the referee for her/his helpful suggestions for the improvement of this work.

\section{REFERENCES}

[1] S. Ebrahimi Atani The ideal theory in the quotients of semirings, Glas. Mat. Ser. III 42(62) (2007), 301-308.

S. E. Atani

Department of Mathematics

University of Guilan

Rasht

Iran

E-mail: ebrahimi@guilan.ac.ir

S. D. P. Hesari

Department of Mathematics

University of Guilan

Rasht

Iran

E-mail: saboura_dolati@yahoo.com

M. Khoramdel

Department of Mathematics

University of Guilan

Rasht

Iran

E-mail: mehdikhoramdel@gmail.com

Received: 21.7.2016.

Revised: 16.9.2016. 